\title{
O GÊNERO PARACALAMITES ZALESSKY 1927, NA TAFOFLORA DE ITAPEVA, CARBONÍFERO SUPERIOR DO SUBGRUPO ITARARÉ, GRUPO TUBARÃO, SUDOESTE DO ESTADO SÃO PAULO, BRASIL
}

\author{
Ana Paula ZAMPIROLLI \\ Mary E. BERNARDES-DE-OLIVEIRA
}

\begin{abstract}
RESUMO
A tafoflora de Itapeva (SP) é parte integrante da Tafoflora A da sucessão neopaleozóica da bacia do Paraná. Caracteriza-se por ser uma flora gondvânica pré-glossopterídeas, neocarbonífera, composta de esfenópsidas, pteridófilas (filicópsidas ou progimnospermópsidas ou pteridospermópsidas e gimnospermópsidas).

O morfogênero Paracalamites aparece como elemento componente dessa tafoflora com três espécies: P. australis Rigby; P. levis Rigby e P. montemorensis Millan. Para as três formas específicas são propostas emendas à diagnose com base nesse material e naquele revisado do Museu Nacional da Universidade Federal do Rio de Janeiro.
\end{abstract}

Palavras Chaves: Paracalamites, esfenópsidas, Itapeva, Subgrupo Itararé, Gondvana Brasileiro

\section{ABSTRACT}

The Itapeva (SP) taphoflora is part of Taphoflora A of the Upper Paleozoic Succession of the Paraná Basin. It is a Late Carboniferous Gondwanan Pre-Glossopterid Flora, composed of: sphenopsids, pteridophylles (or progymnospermopsids) and gymnospermopsids.

In this taphoflora the morphogenus Paracalamites is represented by three species: P. australis Rigby; P. levis Rigby and P. montemorensis Millan. It is proposed to emend. the diagnoses of these species based on the present materials and on a revision of specimens in the National Museum of the Federal University of Rio de Janeiro.

Keywords: Paracalamites, Sphenopsids, Itapeva, Itararé Subgroup, Brazilian Gondwana

\section{INTRODUÇÃO}

Com o estudo dos elementos referidos ao gênero esfenopsida Paracalamites Zalessky dáse prosseguimento ao levantamento dos integrantes da tafoflora neocarbonífera de Itapeva, desenvolvido dentro do projeto temático "Levantamento da composição e sucessão paleoflorísticas do Neocarbonífero-Eopermiano, Grupo Tubarão, no Estado de São Paulo". O estudo e a revisão dessa tafoflora, que vêm sendo realizados, dentro do referido projeto, por ZAMPIROLLI et al. (1999); ZAMPIROLLI \& BERNARDES-DE-OLIVEIRA (1999) e ZAMPIROLLI et al. (no prelo), consti- tuem, em sua síntese, tema da dissertação de mestrado, em desenvolvimento pela primeira autora (A.P.Z.).

\section{MATERIAL ESTUDADO}

Os fitofósseis constituintes da tafoflora de Itapeva, aqui estudados, são provenientes da entrada da mina de carvão abandonada da fazenda Santa Marta, bairro Guarizinho, situado na área setentrional do Município de Itapeva, região sudoeste do Estado de São Paulo (Fig. 1). 


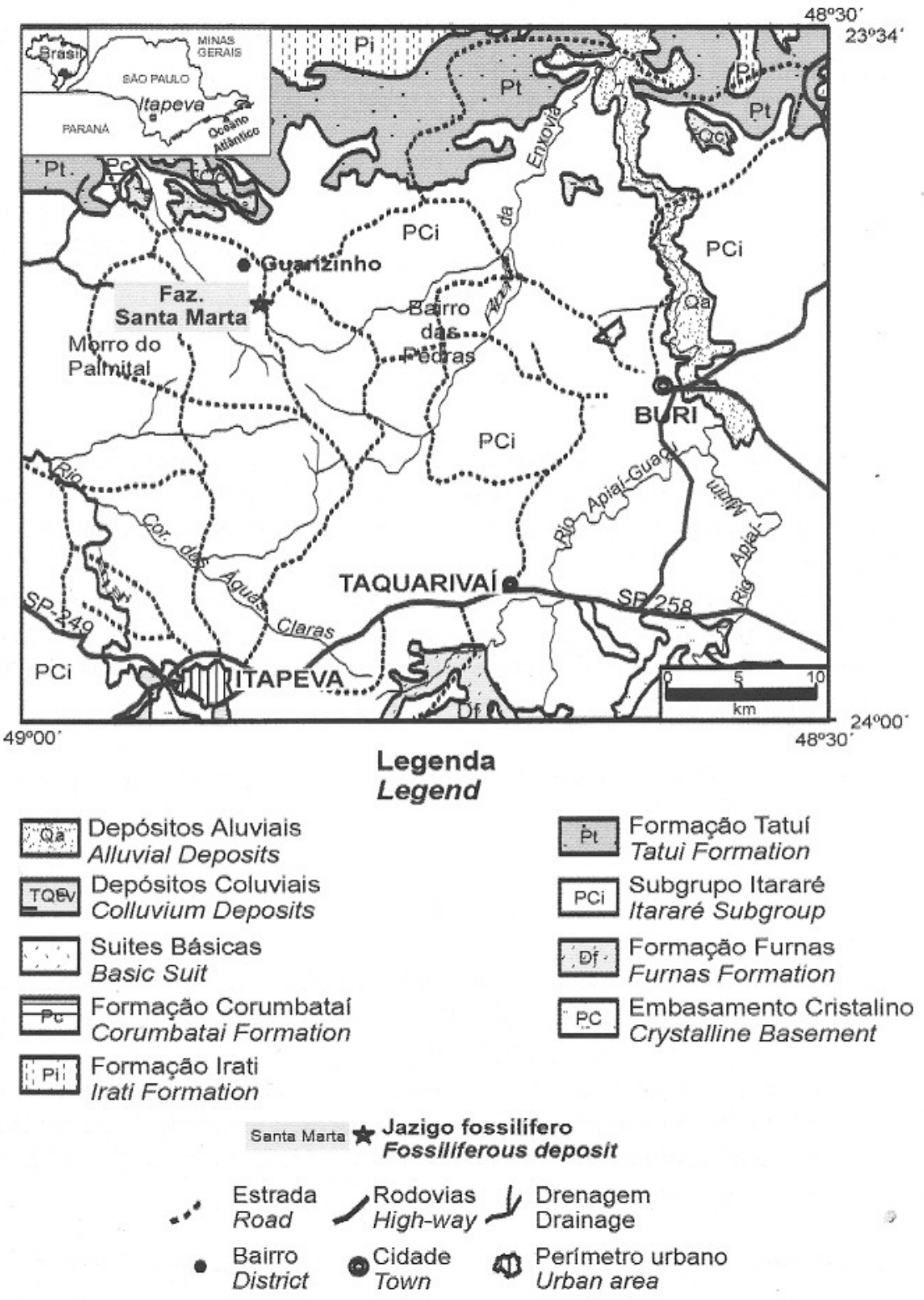

FIGURA 1 - Mapa geológico e de localização do afloramento fitofossilífero fazenda Santa Marta, região de Itapeva (SP) (Modificado de Silva, 1994).

Tratam-se de impressões delicadas e pouco coloridas de caules, folhas e sementes, abundantemente acumuladas e superpostas em meio a material detrítico síltico-argiloso, marrom-claro.

O material estudado compreende alguns espécimes que fazem parte da Coleção Científica do Laboratório de Paleontologia Sistemática do IGc/ USP, estando depositados sob os números GP/3T$2264 ; 2265 ; 2266 ; 2267$, enquanto outros fazem parte da Coleção Científica do Museu Nacional/UFRJ onde estão catalogados sob os números $1970 \mathrm{~Pb}$; $1977 \mathrm{~Pb} ; 1979 \mathrm{~Pb} ; 1981 \mathrm{PbA} ; 1989 \mathrm{~Pb}$ e $1991 \mathrm{~Pb}$.

Os da primeira coleção foram coligidos pelas autoras e os da segunda coleção, pelo paleobotânico Dr. J.H. Millan.

Foram observados, fotografados, mensurados e desenhados sob estereofotomicroscópio C. Zeiss, provido de câmara clara, do Laboratório de Paleobotânica do IGc/USP.

\section{POSICIONAMENTO ESTRATIGRÁFICO DA TAFOFLORA}

A tafoflora de Itapeva constitui parte integrante da Tafoflora A, dentro da sucessão paleoflorística proposta por RÖSLER (1978) para 
a bacia do Paraná, correspondendo a uma flora gondvânica pré- glossopterídeas.

A ocorrência, considerada neocarbo-nífera por seu conteúdo palinológico (SOUZA et al. 1993, 1997 e BERNARDES-DE-OLIVEIRA et al. 1999), tem posição estratigráfica mediano-basal no Subgrupo Itararé, dentro de um contexto regional de sedimentação glácio-flúvio-deltáica, sendo localmente sugestiva de lagunar/deltáica, sob clima peri- ou interglacial, por sua seção colunar, tipo de vegetação e conteúdo palinológico, conforme ZAMPIROLLI et al. (no prelo).

\section{ESTUDOS PRÉVIOS}

A tafoflora da fazenda Santa Marta foi descoberta e registrada por MILLAN et al. (1982). Posteriormente, numa série de trabalhos, MILLAN (1987a, 1987b, 1989, 1991a, 1991b, 1993, 1995a e 1995b) identificou vários componentes da tafoflora chegando ao seguinte quadro:

\begin{tabular}{|l|l|}
\hline Esfenópsidas & $\begin{array}{l}\text { Paracalamites australis } \\
\text { Sphenophyllum } \text { cf. S. churulianum } \\
\text { S. sp. }\end{array}$ \\
\hline Pteridófilas & $\begin{array}{l}\text { Botrychiopsis plantiana } \\
\text { Nothorhacopteris sp. }\end{array}$ \\
\hline Gimnospermópsidas & $\begin{array}{l}\text { Cordaites } \text { cf. C. } \text { spathulata } \\
\text { Cordaites sp. } \\
\text { Cordaicarpus zeilleri } \\
\text { Samaropsis itapevensis }\end{array}$ \\
\hline
\end{tabular}

ZAMPIROLLI et al. (1999a e no prelo) têm acrescentado aos elementos esfenofíticos constituintes dessa tafoflora: Koretrophyllites sp.

Agora uma revisão do morfogênero Paracalamites é elaborada.

\section{SISTEMÁTICA}

Divisão Sphenophyta

Classe Sphenopsida

Ordem Equisetales

Gênero Paracalamites Zalessky 1927

O morfogênero Paracalamites Zalessky abriga grande variedade de caules descritos na literatura paleobotânica do Neopaleozóico, conforme RIGBY (1966a e b). Esses caules, com distribuição geográfica entre Angara e Gondwana, são encontrados nas ocorrências gondvânicas em conexão orgânica com Phyllotheca, Schizoneura,
Raniganjia, Sphenophyllum, Stellotheca, Umbellaphyllites ou associados a diversos outros tipos de verticilos foliares de esfenópsidas. Apresentam sempre as mesmas características principais: caules e rizomas articulados, entrenós mais longos ou menos longos que largos, tendo nos contra-moldes medulares, feixes vasculares e áreas interfasciculares opostos, ou seja, normalmente não-alternos na passagem da linha dos nós, dicotomizando-se e, em alguns casos, recombinando-se (ver RIGBY 1969, Fig. 1).

Os espécimes caulinares articulados desprovidos de folhas, provenientes da tafoflora Santa Marta, não podem ser atribuídos com segurança a um único gênero provido de verticilo foliar de esfenópsida, dado serem igualmente encontrados associados a Koretrophyllites e a Sphenophyllum.

No Subgrupo Itararé, são registradas ocorrências de três espécies do gênero Paracalamites Zalessky: P. australis, P. levis e P. montemorensis. Freqüentemente, ocorre uma dúvida na identificação de alguns espécimes em uma ou em outra espécie. Para contornar esse problema, tentou-se estabelecer critérios mais acurados na distinção das três espécies levando em consideração as diagnoses originais (P. australis Rigby, RIGBY 1966a: 123, Est. 32, fig 19 e Est. 33, figs 20 e 23 e P. australis Rigby emend. Rigby, RIGBY 1969: F2-F7, Est. 1, figs. 1 - 9; Est. 2, figs. 6-8; Est. 3, figs. 4-8 e Fig. texto 1 e 2 ; P. montemorensis Millan, MILLAN 1977:201-202, Est. I, figs. 10-15 e P. levis Rigby, RIGBY 1966a:125 - 126, Est. 33, figs. 21 - 22) e as medidas dos respectivos espécimes. Dentre esses critérios, os que se mostraram mais funcionais e válidos foram os referentes à relação morfométrica entre largura e comprimento dos entrenós. Essa relação morfométrica (largura/comprimento do entrenó), pelo que se observa, permanece constante, seja ela obtida na região apical, mediana ou basal de caules da mesma espécie, ainda que essas dimensões possam variar. É, assim, proposto aqui se observar um intervalo mais ou menos fechado de variação na relação morfométrica largura / comprimento do entrenó, relação essa diagnóstica para cada espécie.

\begin{tabular}{|l|l|}
\hline \multicolumn{1}{|c|}{ Espécies } & \multicolumn{1}{|c|}{$\begin{array}{c}\text { Proporções de largura/ } \\
\text { comprimento do entrenó }\end{array}$} \\
\hline Paracalamites australis & $\mathrm{y} / \mathrm{c}=1: 2 \mathrm{ou}>2$ \\
\hline Paracalamites montemorensis & $\mathrm{y} / \mathrm{c}=1: 1-1: 1,99$ \\
\hline Paracalamites levis & $\mathrm{y} / \mathrm{c}=1: 0,99 \mathrm{ou}<0,99$ \\
\hline
\end{tabular}

Determinando-se as proporções, notase que, em $P$. australis, o entrenó é substancial- 


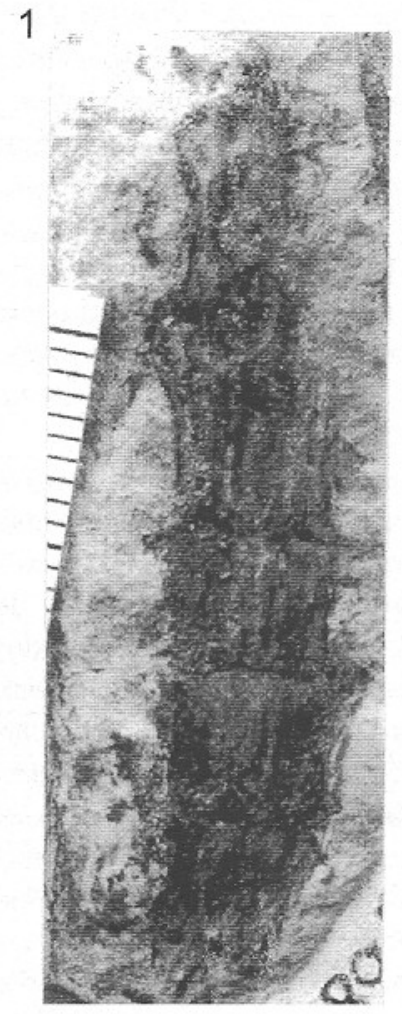

2

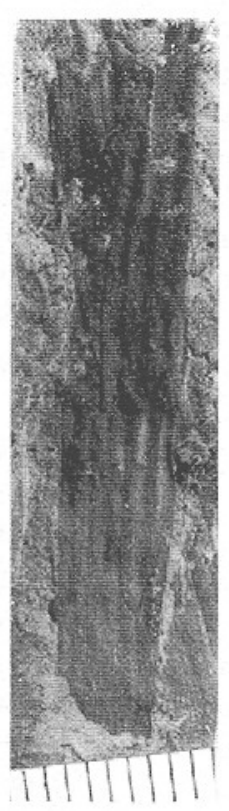

5

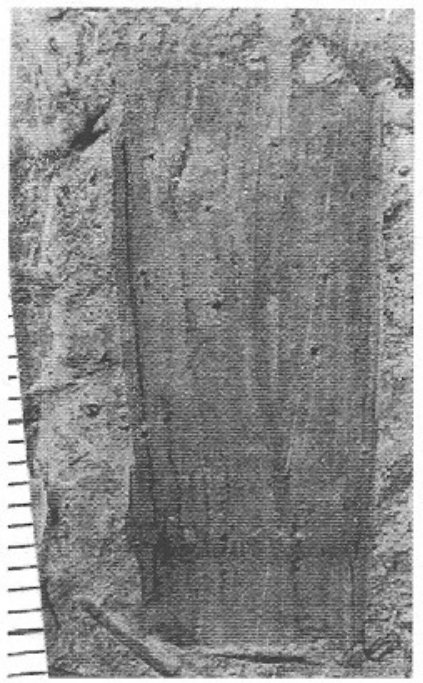

3
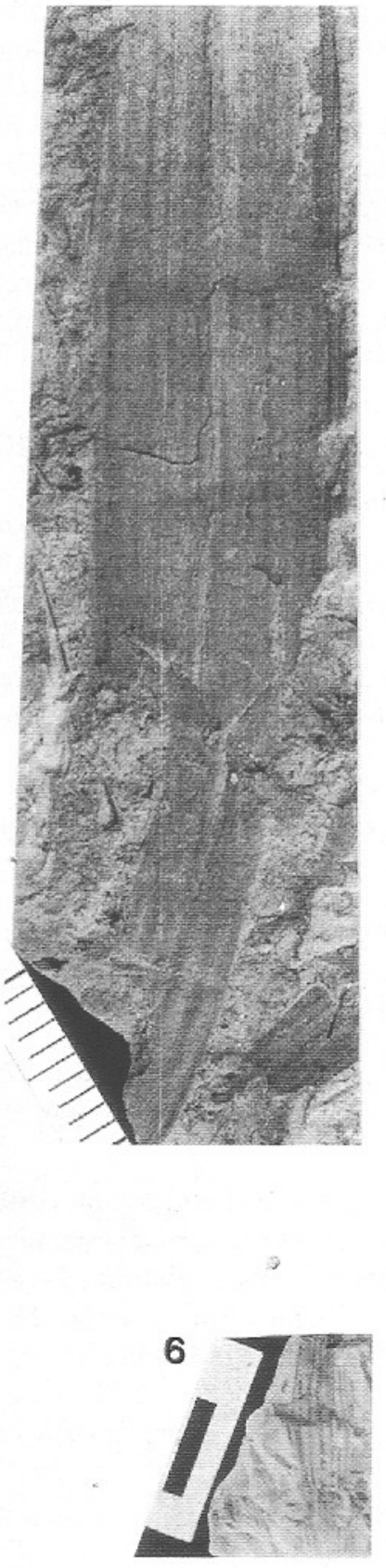

\section{ESTAMPA I}

Figs. 1 e 3 - Paracalamites levis Rigby, 1966 nov. emend. . 1 Col. MN/UFRJ, n $1989 \mathrm{~Pb}$ e 3, Col. MN/UFRJ nº $1981 \mathrm{PbA}$ - Figs. 2 e 5 - Paracalamites montemorensis Millan, 1977 nov. emend.. 2, Col. MN/UFRJ, n $1991 \mathrm{PbA}$ e 5, Col. MN/UFRJ, n 1981 PbB - Fig. 4 - Paracalamites australis Rigby, 1966. Col. MN/UFRJ, nº 1977 Pb. Fig. 6 - Paracalamites sp. Col. IGc/USP, GP/3T nº 2265. 
mente mais longo que a largura do caule; $P$. montemorensis possui entrenós iguais a até quase duas vezes a largura do caule e, em $P$. levis, os entrenós são de comprimento mais curto que a largura do caule.

Adotando-se então esse critério registra-se na tafoflora Santa Marta, a presença das três espécies do Gênero Paracalamites, conforme segue sua descrição abaixo.

5.1 Paracalamites australis Rigby 1966 a emend. Rigby 1969 nov. emend.

$$
\text { (Est. I, Fig. 4) }
$$

P. australis Rigby RIGBY 1966a. Palaeontographica B118:123-124, Pl. 32, fig. 19. Procedência: Grupo Collie, Bacia de Collie e Irwin Coal Measures, Bacia de Perth - Austrália Ocidental.

Sinonímia:

ver RIGBY (1969: F2 - F3) completando com BERNARDES-DE-OLIVEIRA (1977: 79-80) e 1972 - P. australis Rigby. MILLAN, p.50-56, Est. VI, figs. 4,5 e 6 e Est. VII, fig. 2, non Est. VI, figs. 3,7 e 8 .

1972 - P. montemorensis Millan. MILLAN, p. 58 61, Est. VII, figs. 6 e 9 non Est. VII, figs, 4, 5, 7 e 8. 1975 - P. australis Rigby. MILLAN, p. 3.

1977 - P. australis Rigby. MILLAN, p. 196 - 200, Est. I figs. 2, 5, 7 e 8, non Est. I, figs. 1, 3, 4 e 6. 1977 - P. montemorensis Millan. MILLAN, p. 201 - 202, Est. I, figs. 12 e 15 non Est. I, figs, 10, 11,13 e 14 .

1991b - P. australis Rigby. MILLAN, p. 239-243, Est.II, figs. 1, 2 e 3, non Est.I, figs. 5 e 6 e Est. II, figs. 4 e 5 .

Emended Diagnosis from RIGBY 1969 with n. emend.: "Articulate stem having ribs of pith cast opposite at nodes; ribs alternate only to accomodate changes in number of ribs between adjacent internodes. Rib vascular bundles continuous from one internode to the next without break; also vascular bundles dichotomize and recombine with vascular bundles branch of adjacent ribs at nodes. Ribs and furrows sometimes finely striated. Ribbing on node of external cast weak. Internodes substantially longer than stem diameter (1:2 or $>2)$, except in thick truncks".

Espécimes identificados: MN/1970Pb, 1977Pb e $1979 \mathrm{~Pb}$, depositados na Coleção Científica do Museu Nacional/UFRJ, procedentes da Fazenda Santa Marta, bairro Guarizinho, Itapeva (SP).

Descrição: São três impressões de fragmentos de caule articulado, com nós e entrenós desprovidos de ramos, folhas e estruturas reprodutivas.Tratase de moldes medulares apresentando sulcos ou caneluras correspondentes aos feixes vasculares proeminentes.

Os feixes vasculares e áreas interfasciculares paralelos, espaçados de $0,4 \mathrm{~mm}$ - 0,5mm, são contínuos ou, raramente, alternos na passagem pela linha nodal e, freqüentemente, estriados longitudinalmente. As áreas interfasciculares são, geralmente, mais rasas que os feixes e com superfície apenas ondulada.

O espécime $1970 \mathrm{~Pb}$ apresenta comprimento de $22 \mathrm{~mm}$ e largura de $3 \mathrm{~mm}$. exibindo apenas um nó fracamente marcado. Os entrenós apresentam comprimento de $10 \mathrm{~mm}$ enquanto a largura é de $3 \mathrm{~mm}$ (1:3,33). O número de costelas sobre o lado visível é $20 . \mathrm{O}$ espécime $1079 \mathrm{~Pb}$ apresenta comprimento preservado de $25 \mathrm{~mm}$ e largura de $10 \mathrm{~mm}$, tendo nós fracamente marcados, às vezes, quase imperceptíveis. O comprimento de cada entrenó é maior que $18 \mathrm{~mm}$ e sua largura de $9 \mathrm{~mm}$ (1:2). O espécime $1977 \mathrm{~Pb}$ apresenta uma proporção de 1:2,3 entre a largura e o comprimento do entrenó.

Comparação: MILLAN (1991b) havia identificado e descrito como Paracalamites australis os espécimes provenientes de Santa Marta e depositados no Museu Nacional sob os números 1970Pb, $1977 \mathrm{~Pb}, 1979 \mathrm{~Pb}, 1981 \mathrm{PbA}, 1981 \mathrm{PbB}, 1989 \mathrm{~Pb}$, $1991 \mathrm{~Pb}$.

Examinando e fazendo a morfometria de todos os espécimes dessa tafoflora atribuíveis ao gênero Paracalamites, depositados nas coleções científicas do Museu Nacional/UFRJ e do IGc/ USP verificou-se que apenas três espécimes do Museu Nacional (1970Pb, 1977Pb e 1979Pb) figurados e descritos por MILLAN (1991a , Est. II, figs. 1 e 3) podem ser identificados como possuidores dos seguintes caracteres: caules articulados com costelas geralmente opostas nos nós, presença de estriações nas costelas e sulcos, feixes vasculares que se ramificam e se recombinam nas costelas adjacentes dos nós, apresentando entrenós substancialmente mais longos que o diâmetro do caule $(1: 2$ ou $>2)$, exceto nos caules mais espessos. Todos esses caracteres são considerados aqui diagnósticos de P. australis Rigby 1966 emend. Rigby 1969 nov. emend.

Segundo esses critérios, os demais espécimes foram remanejados para as duas outras espécies apresentadas a seguir.

Discussão: Embora a continuidade dos feixes 
vasculares na passagem da linha nodal seja a feição dominante, é possível observar uma alternância dos feixes, num ponto ou outro dessa linha. Em geral, esse fato está associado a um ligeiro aumento do número de feixes de um entrenó para o adjacente, conforme observado por RIGBY (1969: F3). Isso pode conferir à linha nodal um aspecto em zigue-zague, semelhante ao observado no subgênero Mesocalamites Himer 1927, do Namuriano - Westfaliano A das floras Euroamericana e Angárica.

Contudo, dadas: a) a preservação precária dos espécimes estudados; b) a distinção imprecisa, muitas vezes, entre Paracalamites e Mesocalamites, conforme BOUREAU (1964: 244); c) a distribuição geográfica distinta; d) a ausência na assembléia fitofossilífera de aparelhos reprodutivos de tipos que ocorrem em Mesocalamites e e) a maior freqüência de feixes vasculares contínuos; prefere-se manter essas formas sob o morfogênero Paracalamites e sob a espécie $P$. australis.

Há que se observar, entretanto, que os espécimes de $P$. australis provenientes de Itapeva, são de dimensões, sensivelmente, menores que os espécimes australianos dessa espécie, contudo, as proporções são mantidas.

5.2 Paracalamites levis Rigby 1966a nov. emend. (Est. I fig.1 e 3)

Sinonímia:

1966a - P. levis Rigby. RIGBY, p. 125-126, Pl. 33, figs. 22 e 23.

1972 - P. cf. P. levis Rigby. MILLAN, p. 56, Est. VII, fig. 3.

1972 - P. australis Rigby. MILLAN, p. 51, Est. VI fig. 8.

1975 - P. cf. P. levis Rigby. MILLAN, p.3.

1977 - P. cf. P. levis Rigby. MILLAN, p. 200, Est. I, figs. 6 e 9.

1977 - P. australis Rigby. MILLAN, p. 198, Est. I, fig. 6.

1991b - P. australis Rigby. MILLAN, p. 239-243, Est. I, fig. 5 e Est. II, fig.4 (1981PbA - non, 1981 PbB).

Emended diagnosis of RIGBY (1966 a: 125-126): "Slender articulate stem (diameter minor or equal $10 \mathrm{~mm}$ ) having ribs of pith cast opposite at nodes; when number of ribs increasing at nodes, ribs may alternate. Nodes strongly or faintly defined, tubercles absent. Internodes shorter in length than stem diameter $(\mathrm{d}: \mathrm{l}=1: 0.99$ or $1:<0 . .99)$ pith narrowing gradually without bulbous swelling towards base, and without sharp decrease in length of internode. Foliage and fructification unknown". Espécimes identificados: GP/3T-2266 e GP/3T2267 (Col. IGc/USP), $1981 \mathrm{PbA}$ e $1989 \mathrm{~Pb}$ ( Col. MN/UFRJ).

Descrição: São quatro impressões de fragmentos caulinares articulados, com nós e entrenós desprovidos de ramos, folhas e estruturas reprodutivas. Apresentam comprimento da ordem de 24 - 65mm e largura variando entre $8-62 \mathrm{~mm}$. Observam-se de 2 - 10 nós, forte ou fracamente marcados. Tubérculos estão ausentes. Os espécimes GP/3T-2266 e 1989Pb, possíveis moldes caulinares externos apresentando costelas ou cristas (= feixes vasculares) paralelas, proeminentes, espaçadas de 0,4 - 0,5mm, contínuas ou alternadas na passagem da linha nodal. Os outros dois espécimes seriam moldes medulares, apresentando feixes vasculares paralelos, na forma de sulcos, com espaçamento similar, também contínuos raramente alternos na região nodal. Os feixes vasculares e áreas interfasciculares são, freqüentemente, estriados longitudinalmente.

Os entrenós são mais largos que longos variando de 3,2 - 10,5mm de comprimento por 5 $12 \mathrm{~mm}$ de largura, numa proporção média de largura por comprimento de 1:0,57 - 1:0,91.

O número de costelas por feixes vasculares sobre o lado visível é estimado em 8 a 15. Geralmente, áreas interfasciculares rasas ou ligeiramente elevadas e largas $(0,4 \mathrm{~mm})$ estão associadas a feixes fundos, largos $(0,6 \mathrm{~mm})$ e estriados.

Comparação: Ao comparar os espécimes GP/3T2266 e 2267 da Coleção do IGc/USP, com os espécimes 1981PbA e 1989Pb da Coleção do Museu Nacional/UFRJ, coletados e identificados, como P. australis por MILLAN (1991b, Est. I, fig. 5 e Est. II, fig. 4) verifica-se que são, praticamente, idênticos em sua morfologia geral e inclusive quanto à proporção das medidas largura por comprimento do entrenó (1: 0,99 ou < 0,99). Essas característícas são consideradas diagnósticas para P. levis Rigby nov. emend. .O espécime GP/3T 2266 assemelha-se muito ao holótipo de $P$. levis figurado por RIGBY (1966a, Pl. 33, fig. 21) proveniente de Collie Group, Austrália Oriental.

Também o número de costelas sobre a superfície visível dos espécimes brasileiros (cerca de 8) assemelha-se ao dos espécimes de RIGBY (1966a) variando de 8 a 11.

Discussão: RIGBY (1966a e 1969), ao definir os 
caracteres diagnósticos de $P$. levis, afirmou que a largura ou diâmetro do entrenó é maior que o comprimento do entrenó.

Procurando definir melhor essa proporção em contraposição àquelas de $P$. montemorensis Millan e $P$. australis Rigby é que se considera aqui a referida proporção $(1: 0,99$ ou $<0,99)$ e adiciona-se esse caráter à diagnose. Os espécimes de Coleção MN/UFRJ, provenientes de Itapeva; 1989Pb (MILLAN 1991b, Est. I, fig. 9) e 1981PbA (MILLAN, 1991b, Est. II, fig. 4A) identificados como $P$. australis são aqui colocados na sinonímia de $P$. levis por suas características diagnósticas.

\subsection{Paracalamites montemorensis Millan 1977 nov. emend.}

Sinonímia:

$$
\text { (Est.I, figs. } 2 \text { e 5) }
$$

1941 - Phyllotheca sp. Read. READ, 12: 90, Est. 3 , figs. 4 e 5.

1972 - Paracalamites montemorensis Millan. MILLAN, p.58, Est. VII, figs. 4, 5, 7 e 8 non Est. VII, figs. 6 e 9.

1972 - P. australis Rigby. MILLAN, p. 50-56, Est VI, figs. 4, 5 e 6 e Est. VII, fig. 2.

1975 - P. montemorensis Millan. MILLAN, p. 3. 1977 - P. montemorensis Millan. MILLAN, p. 201, Est. I, figs. 10, 11, 13 e 14 non Est. I, figs. 12 e 15. 1977 - P. australis Rigby. MILLAN, p. 196, Est. I, figs. 1, 3, 4 e 5 non Est. I, figs. 2, 7 e 8.

1991b - P. australis Rigby. MILLAN, p. 239-243, Est. I, fig. 6 , Est. II, figs. 4B e 5.

Emended Diagnosis: "Articulate stems, having striated, closed narrow ribs of pith cast, with dense aspect, opposed at nodes, having internodes longer than the stem diameter $(1: 1-1: 1,99)$. Foliage and fructification unknown.

Espécimes identificados: $1981 \mathrm{PbB}$ e $1991 \mathrm{PbA}$ (Col. MN/UFRJ) e GP/3T-2264 (Col. IGc/USP). Descrição: São três impressões de fragmentos caulinares articulados, com nós e entrenós, desprovidos de ramos, folhas e estruturas reprodutivas. Possuem comprimento preservado de $20-35 \mathrm{~mm}$ e largura de $6-40 \mathrm{~mm}$. Normalmente, são observados de um a sete nós sobre os fragmentos caulinares preservados. A nitidez desses nós é variada, às vezes são quase imperceptíveis. Os entrenós apresentam largura variando de $3,5-13 \mathrm{~mm}$ e comprimento de 5 a $25 \mathrm{~mm}$, perfazendo uma proporção largura/ comprimento da ordem de 1:1 - 1: 1,99.

Os espécimes são moldes externos de caule, apresentando caneluras estreitas, muito jun- tas, estriadas, numerosas e opostas na passagem da linha nodal. Os tubérculos são ausentes.

Os feixes vasculares e áreas interfasciculares não se alternam na região dos nós.

O número de costelas ou feixes vasculares sobre o lado visível é estimado de 6 a 16.

Comparação: Os exemplares estudados apresentam uma estreita similaridade de características diagnósticas, incluindo-se as proporções largura/ comprimento dos entrenós, com aquelas identificadas em P. montemorensis Millan e provenientes da tafoflora de Monte Mor (SP), figurados em MILLAN (1972, Est. VII, figs. 4, 5, 7 e 8 e 1977, Est. I, figs. 10, 11, 13 e 14).

Discussão: Há que se ressaltar que alguns espécimes provenientes de Monte Mor (SP), atribuídos por Millan a $P$. montemorensis, devem ser sinominizandos em $P$. australis considerados os seus caracteres e medidas. Esses são: MILLAN (1972, Est. VII, fig. 6 e 9 e 1977, Est. I, fig. 12 e 15) .

\subsection{Paracalamites sp.}

\section{(Est. I fig. 6)}

Espécime identificado: GP/3T-2265 (Col. IGc/ USP)

Descrição: Trata-se de molde externo de fragmento caulinar articulado, apresentando apenas um nó. É desprovido de ramos, folhas e estruturas reprodutivas. Apresenta comprimento superior a $13 \mathrm{~mm}$ e largura de $8,6 \mathrm{~mm}$. O nó é bem nítido e não há tubérculos. As costelas são contínuas na passagem da linha nodal, apresentando dicotomias e recombinações raras, correspondentes à redução ou ao aumento do número de costelas e áreas interfasciculares no entrenó seguinte, chegando nesses pontos a sugerir alternância de costelas na passagem nodal.

Discussão e comparação: No espécime GP/3T2265 nenhum entrenó completo foi preservado. Assim, fica impossível determinar com exatidão a proporção largura por comprimento do entrenó. Pelas medidas apresentadas sabe-se que essa proporção é 1:> 1,5. Com essa proporção indefinida e na ausência de outros caracteres diagnósticos, fica-se na dúvida se o espécime poderia ser identificado como P. montemorensis Millan emend. nov. ou como $P$. australis Rigby emend. nov. Nesses termos, prefere-se referí-lo como Paracalamites sp. 


\section{CONSIDERAÇÕES FINAIS}

A identificação na assembléia de Santa Marta das três espécies do morfogênero Paracalamites, encontráveis no Subgrupo Itararé, é um fato inédito, caracterizando bem a diversidade das esfenópsidas naquela área, aproximando um pouco mais sua composição àquela encontrada na tafoflora de Monte Mor, SP.

Elaborando a revisão do material já estudado e com base em novas coletas, estão sendo detectadas formas foliares esfenofíticas dos gêneros Sphenophyllum e Koretrophyllites, associadas a Botrichyopsis, Nothorhacopteris, Noeggeratiopsis, Cordaicarpus, Samaropsis, caules e folhas indeterminadas.

Esses fitofósseis tratam-se de formas muito fragmentadas e delicadas, em sua maioria higrófilas, depositadas sem uma direção preferencial, sugerindo pouco transporte, mas suficiente para fragmentá-las muito, dada a sua natureza frágil, conforme observaram ZAMPIROLLI \& BERNARDES-DE-OLIVEIRA (1999). A pequena quantidade de material detrítico entre as impressões, que se acumulam umas sobre as outras, também é sugestiva de certa autoctonia ou pequeno transporte.

\section{AGRADECIMENTOS}

À Fundação de Amparo à Pesquisa do Estado de São Paulo, pelo apoio financeiro - Projeto Temático Levantamento da composição e sucessão paleoflorísticas do NeocarboníferoEopermiano (Grupo Tubarão) no Estado de São Paulo, FAPESP 97/03639-8.

\section{REFERÊNCIAS BIBLIOGRÁFICAS}

BERNARDES-DE-OLIVEIRA, M.E. 1977 Tafoflora Eogondvânica da Camada Itapeva, Formação Rio Bonito (Grupo Tubarão), SC. Tese de doutoramento - IGc/ Universidade de São Paulo, 2 volumes. 302 p. +36 estampas (inédito).

—; M. E.; ROHN, R.; SOUZA, P.A; RICARDIBRANCO, F.; RÖSLER, O.; IANNUZZI, R.; ZAMPIROLLI,A.P. 1999. Revision of Upper Paleozoic Phytobiostratigraphy Schemes in the Northern Portion of Paraná Basin. XIV ICCP, Pander Society, Can. Paleont. Conf. Calgary. Programme Abstracts. 1999, p. 12.

BOUREAU, E. 1964. Sphenophyta. In: Traité de
Paléobotanique. Paris. Ed. Masson et Cie. T. 3, 554 p., 436 figs.

HIRMER, M. 1927. Handbuch der Paläobotanik Bd. 1. Thallophyta-BryophytaPteridophyta. 708pp. Vlg. Oldenbourg. München und Berlin.

MILLAN, J.H. 1972. Macroflórula Carbonífera de Monte Mor, Estado de São Paulo. Tese de Doutoramento, Inst. Geoc., Univ. São Paulo, 165pp., 12 est. (inédito).

— 1975. Tafoflórula Monte Mor do Estado de São Paulo. Seus elementos e seu significado no Gondwana Inferior do Brasil. Revista Brasileira de Geociências. São Paulo, 5 (1): 1 - 14

1977. Moldes Medulares da Tafoflórula Gondwânica de Monte Mor, Estado de São Paulo. An. Acad. bras. Ci., Rio de Janeiro, 49 (1): 195-204

; ANDRADE, A. B.; DOLIANITI, E. 1982. Uma nova tafoflórula do Eogondwana de Itapeva, SP. An. Acad. bras. Ci., Rio de Janeiro, 54 (4): 753.

- 1987a. Os pisos florísticos do carvão do Itararé do Estado de São Paulo e suas implicações. $X$ CONGRESSO BRASILEIRO DE PALEONTOLOGIA, Rio de Janeiro, 1987, An. 2: 837-857.

-1987b. Descoberta de frondes de Botrychiopsis plantiana no Eogondwana do Município de Itapeva, Subgrupo Itararé do Estado de São Paulo. X CONGRESSO BRASILEIRO DE PALEONTOLOGIA, Rio de Janeiro, 1987, An. 2: 809-829.

— 1989. Sobre as Sphenopsidas da tafoflora do Eogondwana do Município de Itapeva, carvão do Subgrupo Itararé do Estado de São Paulo. An. Acad. bras. Ci., Rio de Janeiro, 61(4): 479.

— 1991a. Novas ocorrências de sementes platispérmicas nos municípios de Itapeva, SP, e Cerquilho, SP, em tafofloras do Grupo Tubarão, Eogondwana da Bacia do Paraná. An. Acad. bras. Ci., Rio de Janeiro, 63(1): 96.

- 1991b. Sobre as Sphenopsidas de tafoflora associada a camadas carbonosas do Subgrupo Itararé, Eogondwana da Bacia do Paraná, no Município de Itapeva, SP, Brasil. An. Acad. bras. Ci., Rio de Janeiro, 63(3): 229-245. 
- 1993. Sobre as cordaitales da Tafoflora Eogondwânica de Itapeva, SP, Subgrupo Itararé da Bacia do Paraná, Brasil. An. Acad. bras. Ci., Rio de Janeiro, 65(2): 213.

— 1995a. Ocorrência de Nothorhacopteris na Tafoflora de Itapeva, SP, Subgrupo Itararé da Bacia do Paraná, Brasil. An. Acad. bras. Ci., Rio de Janeiro, 67(3): 384.

_ 1995b. Novas ocorrências de sementes platispérmicas em tafofloras associadas a camadas carbonosas do Grupo Tubarão, Eogondwana da Bacia do Paraná, no Estado de São Paulo. An. Acad. bras. Ci., Rio de Janeiro, 67 (1): 117-128, 1 estampa.

READ, C. B. 1941. Plantas fósseis do NeoPaleozóico do Paraná e Santa Catarina. Div. Geol. Miner., DNPM, Monogr. 12: 1 - 102

RIGBY, J. F. 1966a. The Lower Gondwana Floras of the Perth and Collie Basins, Western Austrália. Palaeontogr, AbtB, 118 (4-6): 123 - 126, pls. 32 e 33.

— 1966b. Some Lower Gondwana Articulates from New South Wales. SYMPOSIUM ON FLORISTICS AND STRATIGRAPHY OF THE GONDWANALAND. p.48-54 plate 1.

— 1969. Permian Sphenopsids from Antarctica. Contributions to Paleontology, 1968, U.S. Geol. Surv. Proc. Pap., 613F: 1 - 13, pls. $1-3$.

— 1970. The distribution of Lower Gondwana plants in the Paraná Basin of Brazil and Gondwana. SYMPOSIUM, Pretoria, Proc. and Pap., p. 575 - 584..

RÖSLER, O. 1978. The Brazilian eogondwanic floral successions. Bol.IG-USP, São Pau10, 9: 85-91.

SOUZA, P.A.; LIMA, M.R.; SAAD, A.R. 1993. Palinologia dos carvões paleozóicos do Estado de São Paulo. I- O carvão de Buri. Revista do Instituto Geológico, 14(1): 520.

-;-- -- 1997. Palinologia dos carvões paleozóicos do Estado de São Paulo. I- O carvão de Monte Mor. Revista do Instituto Geológico, 18 (1/2): 7-21.

ZALESSKY, M. D. 1927. Flore paléozoïque de la Série d'Angara. Mem. Com. Géol. (n.s.) 174.

ZAMPIROLLI, A.P.; BERNARDES-DE-OLIVEIRA, M.E.C.; SOUZA, P.A. 1999. Levantamento da composição e sucessão paleoflorísticas do NeocarboníferoEopermiano (Grupo Tubarão) no Estado de São Paulo. II-1. Considerações sobre a tafoflora de pré-glossopterídeas de Itapeva, Subgrupo Itararé, Bacia do Paraná, Brasil. XVI CONGRESSO BRASILEIRO DE PALEONTOLOGIA CRATO. CE., agosto, 1999, Resumos p. 127.

--; M.E.C.1999. Ocorrência de cf. Koretrophyllites, sp., na tafoflora do Eogondvana no Município de Itapeva, SP. REUNIÃO ANUAL DA SOCIEDADE BRASILEIRA DE PALEONTOLOGIA, PALEO 99. Univ. Guarulhos, p. 12.

—; M.E.C.; SOUZA, P. A. (no prelo) Ocorrência de esfenófita equisetaleana cf. Koretrophyllites sp., na tafoflora neocarbonífera do Subgrupo Itararé, Bacia do Paraná, no Município de Itapeva, SP. Revista Geociências UnG, Guarulhos, SP.

Endereço dos autores:

- Ana Paula Zampirolli - Programa de Pós-Graduação em Geologia Sedimentar, Instituto Geociências, Universidade de São Paulo e Uni ABC, Santo André, SP. E-mail:zampirolli@uniabc.br.

- Mary E. Bernardes-de-Oliveira - Programa de Pós-Graduação, Universidade Guarulhos, Guarulhos, SP e Programa de Geologia Sedimentar, Instituto Geociências, Universidade de São Paulo, São Paulo, SP. E-mail: maryeliz@usp.br. 